\title{
Association of Lipid Peroxidation/Antioxidant System Activity with Glutathione S-Transferase P1 Polymorphisms in Infertile Men
}

\author{
N. A. Kurashova, PhD, ScD*; B. G. Dashiev, PhD; M. I. Dolgikh, PhD; E. A. Kudeyarova; \\ A. V. Labygina, PhD, ScD; T. A. Bairova, PhD, ScD; L. I. Kolesnikova, PhD, ScD \\ Scientific Centre for Family Health and Human Reproduction Problems \\ Irkutsk, the Russian Federation
}

\begin{abstract}
The purpose of this study was to assess the association of two polymorphic loci Ile105Val/Ala114Val of the GSTP1 gene with the parameters of oxidative stress (OS) in men with infertility.

We retrospectively analyzed the results of a survey of 222 men (mean age of $29.9 \pm 5.3$ years) of infertile couples. A control group of 104 men (30.2 \pm 3.6 years) was formed, consisting of healthy men with realized reproductive function. An analysis of the frequency with which the Ile105Val polymorphism of the GSTP1 gene occurred in men with infertility and in fertile men found statistically significant differences $\left(\chi^{2}=7.487 ; P=0.024\right)$. When comparing the frequency distribution of the genotypes of the Ala114Val polymorphism of the GSTP1 gene in infertile men and fertile patients, no significant differences were found between the groups $\left(\chi^{2}=3.823 ; P=0.14\right)$. In men with infertility, carriers of the heterozygous genotype of the GSTP1 Ile105Val polymorphism presented an increase in reduced glutathione activity by $7 \%(P=0.0004)$, a decrease in glutathione reductase activity by $20 \%(P=0.03)$ in serum, and a decrease in SOD activity by $8 \%(P=0.01)$ in the ejaculate, unlike fertile men with heterozygous polymorphism, who had an increase in the total antioxidant activity of the blood serum by $20 \%(P=0.0001)$ and a decrease in glutathione peroxidase activity by $24 \%(P=0.03)$ in the ejaculate. In men with infertility, carriers of the heterozygous genotype of the GSTP1 Ala114Val polymorphism presented a decrease in $\alpha$-tocopherol concentration by $15 \%(P=0.002)$, an increase in glutathione peroxidase activity by $25 \%(P=0.0004)$ in the blood and a decrease in SOD activity by $7 \%(P=0.01)$ in the ejaculate, unlike fertile men with heterozygous polymorphism, who had an increase in the concentration of serum conjugated dienes by $19 \%$ $(P=0.0001)$ and a decrease in glutathione-S-transferase activity by $32 \%(P=0.03)$ in the ejaculate. Carrier identification of the GSTP1 Ile105Val and Ala114Val polymorphic loci, as well as the determination of the enzymes of the thiol-disulfide system, can be recommended for an additional assessment of the risk of developing reproductive disorders in men. (International Journal of Biomedicine. 2019;9(4):329-333.)
\end{abstract}

Key Words: male infertility $\bullet$ oxidative stress $\bullet$ glutathione-s-transferase $\bullet$ gene polymorphism

\section{Abbreviations}

AOA, antioxidant activity; AOD, antioxidant defense; CDs, conjugated dienes; GPO, glutathione peroxidase; GR, glutathione reductase, GSSG, oxidized glutathione; GST, glutathione-S-transferase; GSTP1, glutathione S-transferase pi 1 gene; GSH, reduced glutathione; LPO, lipid peroxidation; OS, oxidative stress; ROS, reactive oxygen species.

\section{Introduction}

In modern society, the newly formed family is often faced with a situation where the natural desire to reproduce cannot be realized. From $14 \%$ to $20 \%$ of couples of reproductive age suffer from infertility. Male infertility accounts for about $40 \%$ of infertile marriages. Genetic factors in $30 \%-50 \%$ of cases can be a reason for various forms of male infertility. ${ }^{(1-4)}$ The process of spermatogenesis is influenced by a precisely controlled cascade of activation and deactivation of certain genes. Currently, much attention is paid to the study of polymorphic variants of predisposition genes, which, unlike mutations, appear less distinct in the phenotype, but are not always neutral and often lead to the appearance of metabolic products with altered physicochemical 
properties and functional activity parameters. ${ }^{(5,6)}$ The most suitable genetic markers for research are polymorphic variants of biotransformation genes of xenobiotics, the expression of which, unlike other classes of genes, is directly regulated by the effects of environmental factors of a chemical nature. ${ }^{(7,8)}$ Genetic testing in the pre-symptomatic period makes it possible to identify the hereditary tendencies in the development of future diseases in the genome and, based on modern medical experience, to identify ways of their early prevention. In the pathogenesis of diseases of the reproductive organs, nonspecific processes occurring at the cellular level are essential. ${ }^{(9-11)}$

LPO processes play an important part in disrupting the vital activity of cells and molecular mechanisms, which is associated with the formation of free radicals, which damage the structure and function of membranes. ${ }^{(2,6,11)}$ The processes of LPO and antioxidant protection are a single system that provides cellular homeostasis at the optimal level for the body and is one of the regulatory mechanisms of metabolism. The excess of free radicals and OS caused by them, on the one hand, can have a negative effect on spermatogenesis, but on the other hand, the normal functioning of spermatozoa requires the presence of physiological amounts of ROS. ${ }^{(12-14)}$ In excess, ROS can initiate abnormalities in spermatozoa by inducing oxidative damage to cellular lipids, proteins and DNA, which are the mechanisms of the pathogenesis of male infertility. ${ }^{(5)}$ ROS have a negative effect even within physiological concentrations, since they can stimulate premature capacitation and such irreversible events as the acrosome reaction. ${ }^{(13,15)}$ An indispensable companion of spermatogenesis anomalies can also be a disruption of the activity of thiol-dependent ensembles. ${ }^{(16-18)}$ Pathospermia is accompanied by a decrease in the activity of such antioxidant enzymes as GPO and GST and a decrease in the content of GSH in sperm and seminal plasma. ${ }^{(19-24)}$ Based on the above description, the problem with this study is that the same risk factors, depending on the genetic characteristics of men, can either reduce fertility or not. It is the genetically determined features of the functioning of the xenobiotic biotransformation system that make each individual unique in relation to his adaptive abilities. Two genetic variants in glutathione S-transferase pi 1 gene (GSTP1) - Ile105Val (amino acid isoleucine 105 changed to a valine [rs1695]) and Ala114Val (amino acid alanine 114 changed to a valine [rs1138272]) - have been shown to confer altered catalytic activity of GSTP1. ${ }^{(25-27)}$

The purpose of this study was to assess the association of two polymorphic loci Ile105Val/Ala114Val of the GSTP1 gene with the parameters of OS in men with infertility.

\section{Materials and Methods}

We retrospectively analyzed the results of a survey of 222 men (mean age of $29.9 \pm 5.3$ years) of infertile couples. A control group of 104 men (30.2 \pm 3.6 years) was formed, consisting of healthy men with realized reproductive function. All men had a laboratory and clinical examination by an andrologist, including an ultrasonic scan of scrotum and prostate. Macroscopic and microscopic examination of ejaculate was performed in accordance with the WHO recommendations (2010). Patients with the genetic causes of infertility (AZF-deletions, CFTR-mutations, mutational changes of the number of CAG repeats controlled by androgen receptors) were excluded from the study. In serum and ejaculate of the examined men, the content of thiobarbituric acid (TBA)-active products (TBA-AP) was determined by the method of V.B. Gavrilova et al. (1984). The level of retinol and $\alpha$-tocopherol was estimated by the method of R.Ch. Chernyauskene et al. (1984), total antioxidant activity (AOA) according to G.I. Klebanov et al. (1988). The content of reduced glutathione (GSH) and oxidized glutathione (GSSG) was determined by the method of P.Y. Hissin (1976). The activity of GST, GPO and GR was determined using Randox reagents. The concentration of conjugates during the reaction was registered spectrophotometrically with a wavelength of $340 \mathrm{~nm}$ using a BTS-350 spectrofluorophotometer.

DNA samples were genotyped for polymorphisms in the GSTP1 gene. DNA was isolated from venous blood samples using the sorbent method with the certified reagent kit DNASorb-B (Central Research Institute of Epidemiology, Moscow, Russia).

Genotyping for two common variants in the GSTP1 gene, c.313 A $>$ G (Ile105Val, rs1695) and c.341 C $>$ T (Ala114Val, rs1138272) was performed using methods described by Watson et al. ${ }^{(28)}$ Amplification products were detected in $3 \%$ agarose gel; the electrophoresis results were registered and documented with the help of the system of computer gel documentation GelDoc.

The statistical analysis was performed using the software package Statistica 6.1 (StatSoft, USA) and Biostat. Deviation from Hardy-Weinberg equilibrium and differences in allele distributions between the two groups were assessed by $\chi^{2}$ - test and Yates' chi-square test. Two-tailed $P$ values $<0.05$ were considered statistically significant.

\section{Results and Discussion}

It is known that the degree of expression of various isoenzymes of enzymes of the biotransformation system in different organs and systems varies. Unfortunately, data on the functioning of the glutathione system of spermatozoa as a target organ are rare and indicate the importance of this system in implementing such pathological states as patospermia (asthenozoospermia, oligozoospermia, teratozoospermia), as well as secretory and excretory-toxic types of infertility. ${ }^{(16,21,23)}$ Xenobiotic detoxification system enzymes are involved in metabolic reactions aimed at reducing the activity of foreign substances; the deletion polymorphism of the GST gene family can contribute to the formation of reproductive disorders in men. ${ }^{(29,30)}$

An analysis of the frequency with which the Ile 105 Val polymorphism of the GSTP1 gene occurred in men with infertility and in fertile men found statistically significant differences $\left(\chi^{2}=7.487 ; P=0.024\right)$ (Table 1$)$. Men in the control group with proven fertility have a homozygous Ile105Ile genotype in $54 \%$ of cases, while in men with infertility this genotype was observed in $67 \%$ of cases. The heterozygous genotype of the Ile105 Val polymorphism was more common in the group of fertile patients than in patients with infertility 
( $33 \%$ and $28 \%$, respectively). At the same time, fertile patients carry the mutant genotype Vallo5Val (14\%) 2.8 times more often than men with infertility (5\%). Probably the presence of this genotype is not associated with an increased risk of reproductive disorders in men.

\section{Table 1.}

The frequency distribution of the genotypes of the Ile105Val and Ala114Val polymorphisms of the GSTP1 gene in fertile and the infertile men

\begin{tabular}{|c|c|c|c|c|c|}
\hline Polymorphism & Genotype & $\begin{array}{c}\text { Control } \\
n=104\end{array}$ & $\begin{array}{c}\text { Infertility } \\
n=160\end{array}$ & $P$ & $\chi^{2}$ \\
\hline \multirow{3}{*}{$\begin{array}{l}\text { GSTP1 } \\
\text { Ile105Val }\end{array}$} & Ile/Ile & $56(53.8 \%)$ & $107(66.9 \%)$ & \multirow{3}{*}{0.02} & \multirow{3}{*}{7.49} \\
\hline & Ile/Val & $33(31.7 \%)$ & $44(27.5 \%)$ & & \\
\hline & $\mathrm{Val} / \mathrm{Val}$ & $15(14.4 \%)$ & $9(5.6 \%)$ & & \\
\hline \multirow{3}{*}{$\begin{array}{l}\text { GSTP1 } \\
\text { Ala114Val }\end{array}$} & Ala/Ala & $90(86.5 \%)$ & $123(76.9 \%)$ & \multirow{3}{*}{0.19} & \multirow{3}{*}{3.31} \\
\hline & Ala/Val & $13(12.5 \%)$ & $35(21.8 \%)$ & & \\
\hline & $\mathrm{Val} / \mathrm{Val}$ & $1(0.96 \%)$ & $2(1.2 \%)$ & & \\
\hline
\end{tabular}

When comparing the frequency distribution of the genotypes of the Ala114 Val polymorphism of the GSTP1 gene in infertile men and fertile patients, no significant differences were found between the groups $\left(\chi^{2}=3.823 ; P=0.14\right)$. Heterozygous genotypes were found in $21.7 \%$ of the infertile men and $13 \%$ of the fertile men. The Val114 Val genotype was found in 2 patients with infertility and in 1 fertile man. Carriers of the mutant allele Val114 constituted $1 \%$ in both groups.

In men with infertility, carriers of the heterozygous genotype of the GSTP1 Ile105 Val polymorphism presented an increase in GSH activity by $7 \%(P=0.0004)$, a decrease in GR activity by $20 \%(P=0.03)$ in serum, and a decrease in SOD activity by $8 \%(P=0.01)$ in the ejaculate, unlike fertile men with heterozygous polymorphism, who had an increase in the total AOA of the blood serum by $20 \%(P=0.0001)$ and a decrease in GPO activity by $24 \%(P=0.03)$ in the ejaculate (Figure 1).

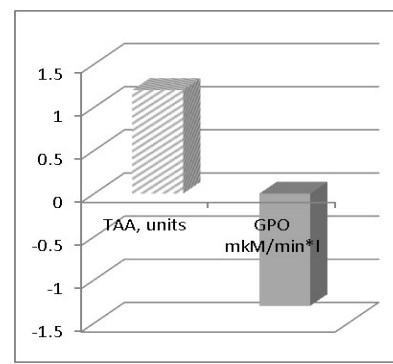

(1)

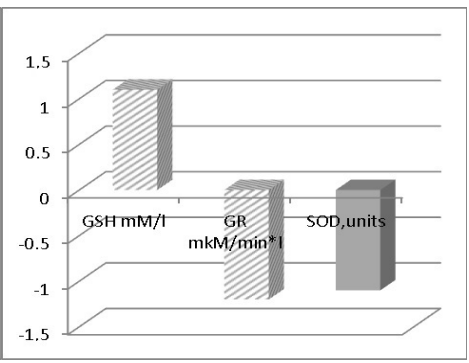

(2)
Fig.1. Statistically significant indicators of the LPO/AOD system in the blood and ejaculate of fertile (1) and infertile (2) men, carriers of the heterozygous genotype of the GSTP1 Ile105Val polymorphism. Here and further, the blood indicators are cross-hatched.

The increased level of the blood total AOA and reduced GPO activity in the ejaculate indicate that antioxidant protection in the group of fertile men is implemented already in the first stages of blocking peroxidation in response to the activation of LPO processes. In men with infertility, the main effect of GSH is realized through participation in the work of antioxidant enzymes. As a substrate for them, GSH acts as a donor of hydrogen atoms for peroxides. SOD performs not only a protective, but also a regulatory function, being a key element in the regulation of constant oxygen concentration. A decrease in SOD activity reduces the inactivation of the superoxide radical, which leads to an increase in the degree of OS. A decrease in the activity of GSH is probably due to its active participation in the GSSG bioregeneration process. A decrease in the power of the antioxidant protection enzyme, in particular one of the components of the thiol-disulfide system, which may not cope with the LPO processes, thereby enhancing them, indicates the development of OS.

In men with infertility, carriers of the heterozygous genotype of the GSTP1 Ala114 Val polymorphism presented a decrease in $\alpha$-tocopherol concentration by $15 \%(P=0.002)$, an increase in GPO activity by $25 \%(P=0.0004)$ in the blood and a decrease in SOD activity by $7 \%(P=0.01)$ in the ejaculate, unlike fertile men with heterozygous polymorphism, who had an increase in the concentration of serum CDs by $19 \%$ $(P=0.0001)$ and a decrease in GST activity by $32 \%(P=0.03)$ in the ejaculate (Figure 2).

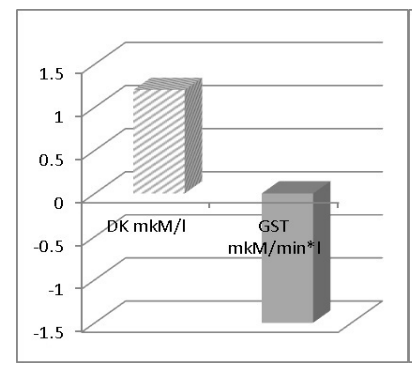

(1)

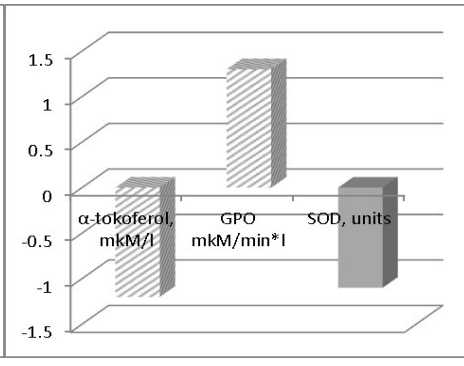

(2)
Fig.2. Statistically significant indicators of the $L P O / A O D$ system in the blood and ejaculate of fertile (1) and infertile (2) men, carriers of the heterozygous genotype of the the GSTP1 Ala114Val polymorphism.

Normally, CDs are involved in regulating membrane permeability, growth rate of organisms, and cell proliferation. GST is the most important multifunctional protein of the ejaculate, since it not only protects against xenobiotics and their metabolites, but also localizes on the surface of spermatozoa and plays the role of a trigger that initiates their interaction with the zonapillucida ligands at the stage of initiation of the acrosomal reaction. Therefore, the determination of GST activity in the ejaculate can be used to determine the fertilizing ability of spermatozoa in men.

Alpha-tocopherol performs several functions, giving a combined antioxidant effect. So interacting with the hydroxyl radical $\mathrm{OH}$, it has a suppressive effect on singlet oxygen. Being a radical trap, $\alpha$-tocopherol is actively involved in blocking the LPO processes and increasing its concentration, possibly due to excessive formation of free radicals in the LPO process. The decrease in the concentration of $\alpha$-tocopherol in the blood of men with infertility, carriers of the heterozygous 
GSTP1 polymorphism (AIa114Val), occurs due to its active participation in metabolic reactions. GPO efficiently interacts with phosphotidylcholine, cholesterol and cholesterol ester hydroperoxides, and is also capable of reducing phospholipid hydroperoxides. It is known that, together with tocopherol, GPO almost completely suppresses LPO in biomembranes. The increased activity of GPO in the blood of men with infertility is likely to be compensatory. SOD activity in the ejaculate of men with infertility is not enough to inactivate reactive oxygen species at the site of their formation, which can lead to diffusion in the medium of tissue macromolecules.

\section{Conclusion}

Thus, it has been found that the glutathione disulfide system is an important component of antioxidant protection, especially against endo- and exogenous metabolites formed during OS. A genetically determined imbalance in the system of glutathione-dependent antioxidant protection determines the LPO activation and contributes to a significant weakening of the metabolic and detoxifying functions of the body. As a result, the susceptibility of cells to the damaging effects of xenobiotics increases significantly; xenobiotics negatively affect spermatogenesis and cause "breakdowns," so subtle and responsive to any change in the external and internal constants of the germ cell formation process. Genetically determined features of the functioning of the xenobiotic biotransformation system make each individual unique in relation to his adaptive capacity - stability or sensitivity to damaging exogenous and endogenous factors. Carrier identification of the GSTP1 Ile105Val and Ala114Val polymorphic loci, as well as the determination of the enzymes of the thiol-disulfide system, can be recommended for an additional assessment of the risk of developing reproductive disorders in men. Evaluation of the real state of reproductive health of the male population and the forecast of its changes in the future is not only an important scientific problem, but also essential for monitoring the reproductive health of the population, as well as for creating new medical programs for its preservation and improvement.

\section{Competing Interests} interests.

The authors declare that they have no competing

\section{References}

1. Galimov SN, Akhmetov RM, Galimova EF, Bairamgulov FM, Bikkulova LR [Molecular aspects of the impact of the Speroton complex on the male fertility in idiopathic infertility]. Urologiia. 2017;(2):88-92.[Article in Russian].

2. Agarwal A, Mulgund A, Sharma R, Sabanegh E Mechanisms of oligozoospermia: an oxidative stress perspective. Syst Biol Reprod Med. 2014;60(4):206-16. doi: 10.3109/19396368.2014.918675

3. Ammar O, Haouas Z, Hamouda B, Hamdi H, Hellara I, Jlali A, et al. Relationship between sperm DNA damage with sperm parameters, oxidative markers in teratozoospermic men. Eur J Obstet Gynecol Reprod Biol. 2018;233:70-75. doi: 10.1016/j. ejogrb.2018.12.003.

4. Kovac JR, Pastuszak AW, Lamb DJ The use of genomics, proteomics, and metabolomics in identifying biomarkers of male infertility. Fertil Steril 2013; 99(4):998-1007. doi: 10.1016/j.fertnstert.2013.01.111.

5. Dorostghoal M, Kazeminejad SR, Shahbazian N, Pourmehdi M, Jabbari A. Oxidative stress status and sperm DNA fragmentation in fertile and infertile men. Andrologia. 2017;49(10). doi: 10.1111/and.12762

6. Darenskaya MA, Kolesnikov SI, Rychkova LV, Grebenkina LA, Kolesnikova LI. Oxidative stress and antioxidant defense parameters in different diseases: ethnic aspects. Free Rad Biol Med. 2018;120(S1):60. doi: 10.1016/j. freeradbiomed.2018.04.199

7. Yarosh SL, Kokhtenko EV, Curnosov MI, Solodiliva MA, Polonikov AV. Joint effect of glutathione S-transferase genotypes and cigarette smoking on idiopathic maleinfertility. Andrologia. 2015; 47(9):980-986. doi:10.1111/ and.12367.

8. Semenova NV, Madaeva IM, Darenskaya MA, Bairova TA, Ievleva KD, Kolesnikova LI Lipid peroxidation and antioxidant system parameters in menopausal women with different glutathione s-transferase M1 genotypes. Free Radical Biology \& Medicine. 2019; 139(S1): S39.

9. Glybochko PV, Alyaev YuG, Chalyy ME, Usacheva OA. [Influence of the glutatione S-transferases T1 and M1 gene polymorphisms on androgenic status and semen quality after surgical treatment of varicocele]. Andrology and Genital Surgery. 2013;14(1): 23-26.[Article in Russian].

10. Semenova NV, Madaeva IM, Darenskaya MA, Bairova TA, Ievleva KD, Kolesnikova LI Antioxidant system activity in asian menopausal women depending on the glutathione s-transferase M1 and T1 genotypes. Free Radical Biology \& Medicine. 2019;139(S1):S39-S40.

11. Agarwal A, Virk G, Ong C, du Plessis SS Effect of oxidative stress on male reproduction. World J Mens Health. 2014;32(1):1-17. doi: 10.5534/wjmh.2014.32.1.1

12. Ahmadi S, Bashiri R, Ghadiri-Anari A, Nadjarzadeh A Antioxidant supplements and semen parameters: An evidence based review. Int J Reprod Biomed (Yazd). 2016;14(12):729-736. 13. Intasqui P, Antoniassi MP, Camargo M, Nichi M, Carvalho VM, Cardozo KH et al. Differences in the seminal plasma proteome are associated with oxidative stress levels in men with normal semen parameters. Fertil Steril. 2015;104(2):292301. doi: 10.1016/j.fertnstert.2015.04.037.

14. Moretti E, Collodel G, Fiaschi AI, Micheli L, Iacoponi F, Cerretani D. Nitric oxide, malondialdheyde and nonenzymatic antioxidants assessed in viable spermatozoafrom selected infertile men. Reprod Bio. 2017;17(4):370-375. doi: 10.1016/j. repbio.2017.10.003.

15. Kolesnikova LI, Kurashova NA, Bairova TA, Osipova EV Activity of components of lipid peroxidation system and antioxidant protection in men with infertility, carriers of non-functional genotypes GSTT1 and GSTM1. Free Radical Biology \& Medicine 2018;120(S1):S72-S73. doi:10.1016/j. freeradbiomed.2018.04.240.

16. Lakpour N, Mirfeizollahi A, Farival S, Akhondi MM, Hashemi SB, Amiriannati N, et al. The association of seminal plasma antioxidant levels and sperm chromatin status with

*Corresponding author: Nadezhda A. Kurashova, PhD, ScD. Scientific Centre for Family Health and Human Reproduction Problems, Irkutsk, the Russian Federation.E-mail: nakurashova@ yandex.ru 
genetic variants of GSTM1 and GSTP1 (Ile105Val and Ala114Val)in infertilemen with oligoasthenoteratozoospermia. Dis Markers. 2013;34(3):205-210. DOI:10.3233/DMA120954.

17. Kolesnikova LI, Kurashova NA, Bairova TA, Dolgikh MI, Ershova OA, Korytov LI, Koroleva NV, Dashiev BG Role of glutathione-s-transferase family genes in male infertility. Bulletin of Experimental Biology and Medicine 2017;163(5): 643-645.

18. Kurashova N, Dolgikh MI, Ershova OA, Gavrilova OA, Osipova EV, Dashiev BG, et al. Associations of polymorphic variants of the biotransformation genes with the components of the glutathione system in men with infertility. International Journal of Biomedicine 2017;7(3):226-230.

19. Kan HP, Wu FL, Guo WB, Wang YZ, Li JP, Huang YQ, et al. Null genotypes of GSTM1 and GSTT1 contribute to male factor infertility risk: A meta-analysis. Fertil Steril. 2013;99(3):690-6. doi: 10.1016/j.fertnstert.2012.10.037.

20. Safarinejad M, DadkhahF,AliAsgari M, Hosseini S, Kolahi AA, Iran-Pour E. Glutathione S-transferase polymorphisms (GSTM1, GSTT1, GSTP1) and male factor infertility risk: a pooled analysis of studies. Urol J. 2012;9(3):541-8.

21. Safarinejad MR, Shafiei N, Safarinejad S. The association of glutathione-S-transferase gene polymorphisms (GSTM1, GSTT1, GSTP1) with idiopathic male infertility. J Hum Genet. 2010;55(9):565-70. doi:10.1038/ jhg.2010.59.

22. Tang K, Xue W, Xing Y, Xu S, Wu Q, Liu R, et al. Genetic polymorphisms of glutathione S-transferase M1, T1, and P1, and the assessment of oxidative damage in infertile men with varicoceles from northwestern China. J Androl. 2012;33(2): 257-63. doi:10.2164/ jandrol.110.012468.

23. Tirumala Vani G, Mukesh N, Siva Prasad B, Rama Devi P, Hema Prasad M, Usha Rani P, Pardhanandana Reddy P. Role of glutathione S-transferase Mu-1 (GSTM1) polymorphism in oligospermic infertile males. Andrologia. 2010;42(4):213-7. doi: 10.1111/j.1439-0272.2009.00971.x 24. Huang XK, Huang YH, Huang JH, Liang JY Glutathione S-transferase P1 Ile105Val polymorphism and male infertility risk: an updated meta-analysis. Chin Med J (Engl). 2017;130(8):979-985. doi: 10.4103/0366-6999.204102.

25. Zimniak P, Nanduri B, Pikuła S, Bandorowicz-Pikuła J, Singhal SS, Srivastava SK, et al. Naturally occurring human glutathione S-transferase GSTP1-1 isoforms with isoleucine and valine in position 104 differ in enzymic properties. Eur J Biochem. 1994;224(3):893-9.

26. Hu X, Xia H, Srivastava SK, Herzog C, Awasthi YC, Ji X, Zimniak P, Singh SV. Activity of four allelic forms of glutathione S-transferase hGSTP1-1 for diol epoxides of polycyclic aromatic hydrocarbons. Biochem Biophys Res Commun. 1997;238(2):397-402.

27. Sundberg K, Johansson AS, Stenberg G, Widersten M, SeidelA, Mannervik B, Jernström B. Differences in the catalytic efficiencies of allelic variants of glutathione transferase P1-1 towards carcinogenic diol epoxides of polycyclic aromatic hydrocarbons. Carcinogenesis. 1998;19(3):433-6.

28. Watson MA, Stewart RK, Smith GB, Massey TE, Bell DA. Human glutathione S-transferase P1 polymorphisms: relationship to lung tissue enzyme activity and population frequency distribution. Carcinogenesis. 1998;19(2):275-80.

29. Xiong DK, Chen HH, Ding XP1, Zhang SH, Zhang JH. Association of polymorphisms in glutathione S-transferase genes (GSTM1, GSTT1, GSTP1) with idiopathic azoospermia or oligospermia in Sichuan, China. Asian J Androl. 2015;17(3):481-6. doi: 10.4103/1008-682X.143737.

30. Kurashova NA, Bairova TA, Dolgikh MI, Dashiev BG, Kolesnikova LI. Features of the processes of lipoperoxidation and antioxidant protection in the pathogenesis of infertility in men of different ethnic groups, carriers of nonfunctional polymorphisms of the GSTT1 and GSTM1 genes. International Journal of Biomedicine. 2019;9(1):31-34. 